\title{
A model of fretting wear in the contact of an axisymmetric indenter and a visco-elastic half-space
}

Cite as: AIP Conference Proceedings 1683, 020040 (2015); https://doi.org/10.1063/1.4932730

Published Online: 27 October 2015

Andrey V. Dimaki, and Valentin L. Popov

\section{ARTICLES YOU MAY BE INTERESTED IN}

Engineering calculation of fracture toughness of materials with chevron-notched specimens AIP Conference Proceedings 1683, 020039 (2015); https://doi.org/10.1063/1.4932729

Meso- and microstructural features of steel 12GBA produced by different methods of thermomechanical treatment

AIP Conference Proceedings 1683, 020037 (2015); https://doi.org/10.1063/1.4932727

Features of plastic deformation and fracture of dispersion-strengthened $\mathrm{V}-\mathrm{Cr}-\mathrm{Zr}$-W alloy depending on temperature of tension

AIP Conference Proceedings 1683, 020042 (2015); https://doi.org/10.1063/1.4932732

\section{Lock-in Amplifiers up to $600 \mathrm{MHz}$}
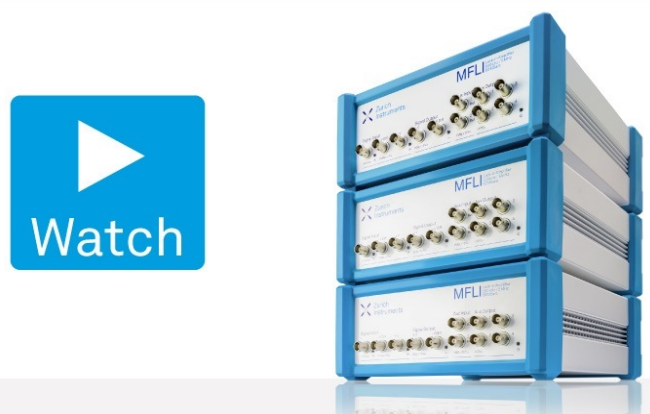


\title{
A Model of Fretting Wear in the Contact of an Axisymmetric Indenter and a Visco-Elastic Half-Space
}

\author{
Andrey V. Dimaki ${ }^{1,2, a)}$ and Valentin L. Popov ${ }^{2,3}$ \\ ${ }^{1}$ Institute of Strength Physics and Materials Science SB RAS, Tomsk, 634055 Russia \\ ${ }^{2}$ National Research Tomsk State University, Tomsk, 634050 Russia \\ ${ }^{3}$ Berlin University of Technology, Berlin, 10623 Germany

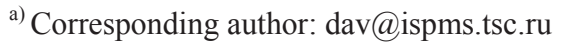

\begin{abstract}
We propose a simple and efficient model of wear of axially symmetric bodies in contact with a visco-elastic foundation based on the method of dimensionality reduction. The results of simulation of wear of a parabolic indenter have been demonstrated. It has been shown that dissipation due to viscosity of a material leads to increase the size of the worn region of an indenter. The noted effect is conditioned with an increase of effective shear modulus of visco-elastic material under sufficiently high velocities of tangential loading. The model can be generalized to a wide range of materials with complex visco-elastic properties.
\end{abstract}

Fretting wear occurs if two bodies are pressed against each other and are subsequently subjected to oscillations with small amplitude. Even if there is no gross slip in the contact, tangential slip occurs at the border of the contact area leading to wear and fatigue. Fretting wear remains an subject of intensive experimental investigation and theoretical simulation for such applications as fretting of tubes in steam generators and heat exchangers [1-3], joints in orthopedics [4], electrical connectors [5], and dovetail blade roots of gas turbines [6, 7] as well as many others. Most theoretical works were concerned with a finite element or boundary element simulations [3]. Even while these simulations provided a complete picture of fretting wear, they still require too much computational time to be implemented as an interface in larger dynamic simulations. In a conventional finite element fretting simulation most of the time is wasted not on the calculation of wear itself but for the solution of the normal and tangential contact problems of a progressively changing profile. In this paper we suggest completing this step using the method of dimensionality reduction [8-10]. This drastically reduces the time of the whole simulation $[10,11]$.

In the paper, we assume that an indenter is absolutely rigid and subjected to wear while a foundation is viscoelastic, in contrast with our previous studies $[10,11]$ where only purely elastic foundations were considered. We again apply the broadly used wear equation stating that the wear volume is proportional to the dissipated energy and inversely proportional to the hardness $\sigma_{0}$ of the worn material. This kind of wear criterion was first proposed by Reye [12], and later justified in detail theoretically and experimentally for abrasive wear [13] and for adhesive wear [14]. The local formulation of this criterion means that the linear change of the three-dimensional profile $I(r)$ is given by the equation

$$
\Delta I(r)=\frac{k}{\sigma_{0}} \tau(r)\left(\Delta u_{x}^{(3 \mathrm{D})}(r)-\Delta u_{x}^{(0)}\right),
$$

where $k$ is the dimensionless wear coefficient, $u_{x}^{(0)}$ is the relative tangential displacement, $u_{x}^{(3 \mathrm{D})}(r)$ is a part of relative tangential displacement due to elastic deformation of a medium, $\tau(r)$ is tangential stress and the symbol $\Delta$ indicates an increment of the corresponding parameter during one step of spatial displacement. No wear occurs in positions where either the tangential stress or the relative displacement is zero.

The main steps of the method of dimensionality reduction (MDR) are the following. Given a three-dimensional profile $z=I(r)$ (see Fig. 1a), we first determine the equivalent one-dimensional profile [8] 


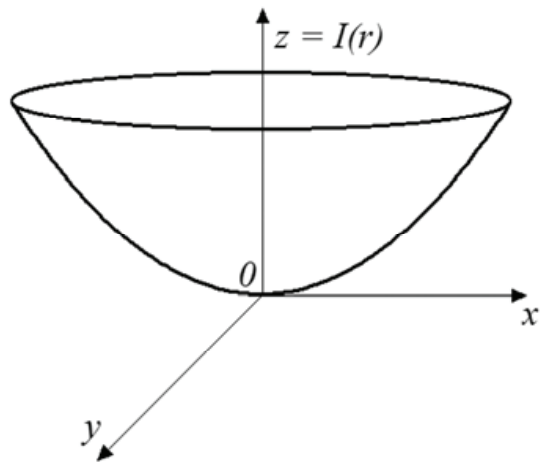

(a)

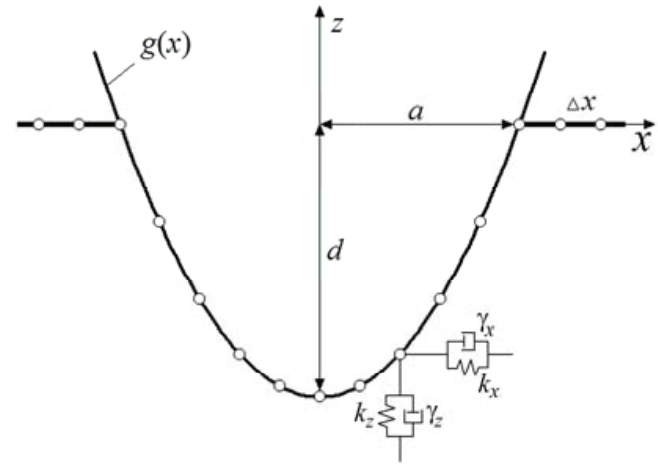

(b)

FIGURE 1. The 3-dimensional body of revolution (a); and the corresponding one-dimensional MDR-transformed profile in a contact with the visco-elastic foundation (only one pair of Kelvin elements has been shown)

$$
g(x)=|x| \int_{0}^{|x|} \frac{I^{\prime}(r)}{\sqrt{x^{2}-r^{2}}} \mathrm{~d} r .
$$

The back transformation is given by the integral

$$
I(r)=\frac{2}{\pi} \int_{0}^{r} \frac{g(x)}{\sqrt{r^{2}-x^{2}}} \mathrm{~d} x .
$$

The profile (3) is pressed to a given indentation depth $d$ into an initially flat foundation consisting of independent elements with spacing $\Delta x$. Note that hereinafter we will consider an indenter of parabolic shape. We assume that the half-space is a non-compressible visco-elastic body having the shear modulus $G$ and viscosity $\eta$ (Kelvin body). In this case, each element of the foundation (see Fig. 1b) will consist of springs with normal and tangential stiffness [8]

$$
k_{z}=4 G \Delta x, k_{x}=\frac{8}{3} G \Delta x
$$

and dampers with normal and tangential damping constants

$$
\gamma_{z}=4 \eta \Delta x, \gamma_{x}=\frac{8}{3} \eta \Delta x
$$

The resulting vertical displacements of springs are given by

$$
u_{z}(x)=d-g(x)
$$

The contact radius $a$ is given by the condition

$$
g(a)=d
$$

If an indenter moves tangentially according to the law $u_{x}(t)=u_{x}^{(0)} \sin \omega t$, where $\omega$ denotes the angular frequency of oscillations, the springs and dampers will be stressed in the tangential direction with the tangential force

$$
f_{x}=k_{x} u_{x}^{(0)} \sin \omega t+\gamma_{x} u_{x}^{(0)} \omega \cos \omega t .
$$

The springs in contact will stick to the indenter until the tangential force achieves the critical value $\mu f_{z}$, where $\mu$ is the coefficient of friction. After this, the tangential force remains constant and equal to $\mu f_{z}$ while the springs begin to slide. The same is valid if the movement starts from an arbitrary stress state of a spring. It either follows the indenter if the tangential force is smaller than the critical one, or it slides, in which case the tangential force is equal to the critical value. Thus, for any incremental change of the tangential displacement at time $t$ the following equations are valid: 


$$
\begin{aligned}
& \Delta u_{x}(x, t)=\Delta u_{x}^{(0)}, \text { if }\left|k_{x} u_{x}(x, t)\right|<\mu f_{z}, \\
& u_{x}(x, t)=\frac{ \pm \mu f_{z}(x, t)+u_{x}(x, t-\Delta t) \gamma_{x} / \Delta t}{k_{x}+\gamma_{x} / \Delta t} \text { in the sliding state, }
\end{aligned}
$$

where $\Delta t$ is a time step of the model. The sign in the last line of this equation depends on the direction of tangential force. By following incremental changes in the indenter position, the absolute tangential displacement can be determined unambiguously at any location and any point in time. The tangential force density is equal to

$$
q_{x}(x)=f_{x} / \Delta x .
$$

Distributions of tangential stresses $\tau(r)$ and displacements $u_{x}^{(3 \mathrm{D})}(r)$ in the initial three-dimensional problem are defined by equations similar to [8]:

$$
\begin{gathered}
u_{x}^{(3 \mathrm{D})}(r)=\frac{2}{\pi} \int_{0}^{r} \frac{u_{x}(x) \mathrm{d} x}{\sqrt{r^{2}-x^{2}}}, \\
\tau(r)=-\frac{1}{\pi} \int_{r}^{\infty} \frac{q_{x}^{\prime}(x) \mathrm{d} x}{\sqrt{x^{2}-r^{2}}}=-\frac{G}{\pi} \int_{r}^{\infty} \frac{u_{x}^{\prime}(x) \mathrm{d} x}{\sqrt{x^{2}-r^{2}}} .
\end{gathered}
$$

The radius $c$ of the stick region will be given by the condition that the absolute value of the tangential force at this point never exceeds the product of coefficient of friction $\mu$ and the normal force $k_{z} u_{z}(c)$ :

$$
\left|\frac{8}{3} G u_{x}^{(0)} \sin \omega t+\frac{8}{3} \eta u_{x}^{(0)} \omega \cos \omega t\right| \leq 4 \mu G(d-g(c)) .
$$

This gives

$$
\frac{8}{3} u_{x}^{(0)} \sqrt{G^{2}+(\eta \omega)^{2}}=4 \mu G(d-g(c))
$$

This equation determines the radius $c$ of the stick-region which will not be worn. The profile in the limiting shakedown state $I_{\infty}(r)$ is given by the same equation

$$
I_{\infty}(r)=\left\{\begin{array}{l}
I_{0}(r) \text { for } 0<r<c, \\
\frac{2}{\pi} \int_{0}^{c} \frac{g_{0}(x)}{\sqrt{r^{2}-x^{2}}} \mathrm{~d} x+\frac{2}{\pi} d \int_{c}^{r} \frac{1}{\sqrt{r^{2}-x^{2}}} \mathrm{~d} x \text { for } c<r<a
\end{array}\right.
$$

as found in [9], where $I_{0}(r)$ is the initial (not worn) profile and $g_{0}(x)$ the corresponding MDR-image.

The results of numerical simulation of fretting wear of a parabolic indenter, pressed to a constant indentation depth $d_{0}$ into a visco-elastic foundation, are shown in Fig. 2. All vertical coordinates are normalized by $d_{0}$ and the horizontal coordinates by $a_{0}$ which represents the initial contact radius between an indenter and foundation.

As one can see, the explicit taking into account of the viscous dissipation (performed by means of introducing a viscosity into the equation for the tangential reaction force in the numerical model) leads to significant changes in the worn profile, which corresponds to the analytical estimate (15). During that, the worn region expands both to the center of the indenter and in the opposite direction, in comparison with a worn profile in contact with a purely elastic half-space. The noted effect is conditioned by the expansion of a slip region due to an increase in the effective shear modulus of visco-elastic material under significantly high velocities of tangential loading.

The influence of parameters of loading on wear in contact with a visco-elastic foundation needs further detailed study, primarily on the influence of amplitude and frequency of tangential oscillations, because of the frequencydependent response of a visco-elastic foundation. Moreover, the normal pressing force acting on the indenter should also produce an effect. In general, the developed model is universal and can be easily generalized for simulation of a wear of bodies with complex visco-elastic properties and arbitrary axially-symmetric geometry. 


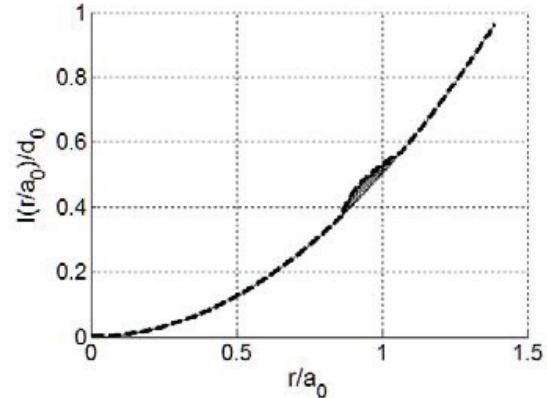

(a)

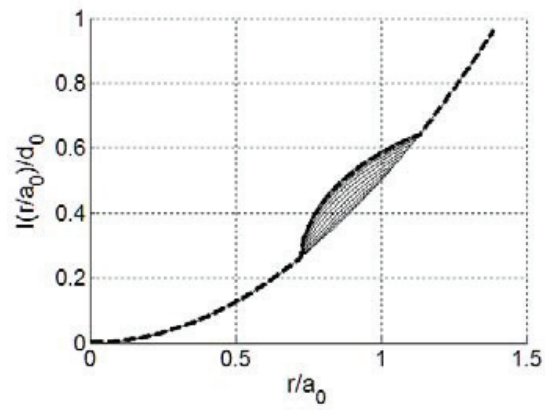

(c)

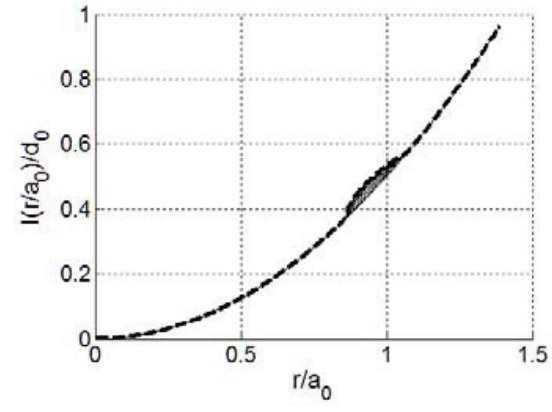

(b)

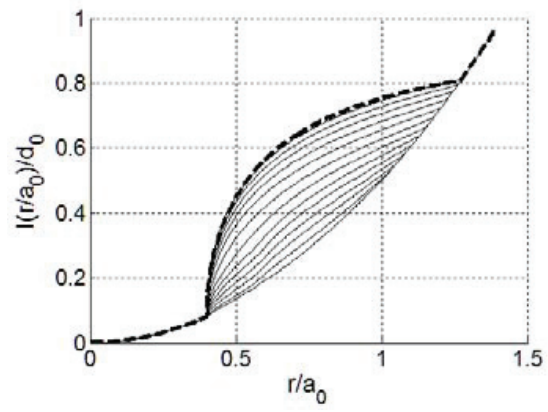

(d)

FIGURE 2. Evolution of the profiles of worn parabolic indenter at different values of the characteristic relaxation time of the material $\Delta t_{\text {relax }}=\eta / G=0(\mathrm{a}), 10^{-4}(\mathrm{~b}), 5 \times 10^{-4}(\mathrm{c}), 10^{-3} \mathrm{~s}(\mathrm{~d})$. Dashed line indicates the analytic estimate (15)

\section{ACKNOWLEDGMENTS}

The authors acknowledge financial support from the Program of Basic Scientific Research of the State academies of sciences for 2013-2020 (Russia).

\section{REFERENCES}

1. P. L. Ko, J. Press. Vessel Technol. 101, 125-133 (1979).

2. N. J. Fisher, A. B. Chow, and M. K. Weckwerth, J. Press. Vessel Technol. 117, 312-320 (1995).

3. C. Y. Lee, L. S. Tian, J. W. Bae, and Y. S. Chai, Tribol. Int. 42, 951-957 (2009).

4. J. P. Collier, M. B. Mayor, R. E. Jensen, V. A. Surprenant, H. P. Surprenant, J. L. MacNamar, and L. Belec, Clin. Orthpaedics Relat. Res. 285, 129-139 (1992).

5. M. Antler, Components Hybrids Manuf. Technol. 8, 87-104 (1985).

6. R. Rajasekaran and D. Nowell, Tribol. Int. 39, 1277-1285 (2006).

7. M. Ciavarella and G. Demelio, Int. J. Solids Struct. 38, 1791-1811 (2001).

8. V.L. Popov and M. Heß, Method of Dimensionality Reduction in Contact Mechanics and Friction (Springer, Berlin, 2015).

9. V. L. Popov, Sci. Rep. 4, 3749 (2014).

10. A. V. Dimaki, A. I. Dmitriev, Y. S. Chai, and V. L. Popov, Int. J. Solids Struct. 51, 4215-4220 (2014).

11. Q. Li, A. E. Filippov, A. V. Dimaki, Y. S. Chai, and V. L. Popov, Phys. Mesomech. 17, $236-241$ (2014).

12. T. Reye, Der Civilingenieur 4, 235-255 (1860).

13. M. M. Khrushchov and M. A. Babichev, Investigation of Wear of Metals (Russ. Acad. Sci., Moscow, 1960).

14. J. F. Archard and W. Hirst, Proc. R. Soc. Lond. A 236, 397-410 (1956). 